\title{
Thyrotropine releasing hormone accelerates wound healing in hyperglycemia inducted mouse fibroblast cell line
}

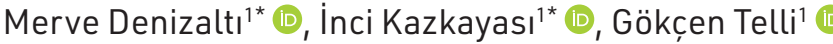 \\ ${ }^{1}$ Hacettepe University, Faculty of Pharmacy, Department of Pharmacology, Ankara, Turkey \\ ${ }^{*}$ Co-first author
}

ORCID IDs of the authors: M.D. 0000-0001-5363-606X; I.K. 0000-0033-1159-9680; G.T. 0000-0003-0028-6769

Cite this article as: Denizalti, M., Kazkayasi, I., \& Telli, G. (2021). Thyrotropine releasing hormone accelerates wound healing in hyperglycemia inducted mouse fibroblast cell line. Istanbul Journal of Pharmacy, 51 (3), 313-318.

\begin{abstract}
Background and Aims: It has been suggested that thyrotropin-releasing hormone (TRH) may have functions beyond its fundamental regulating function. Previous studies have demonstrated that TRH promotes wound healing. We aimed to perform an in vitro study in fibroblasts to assess the role of TRH in wounds that frequently occur in diabetes. Thus, we investigated the effects of TRH in wound healing both under normoglycemic and hyperglycemic-conditions.

Methods: L929 mouse fibroblast cell line was used in the experiments. The cell viability was confirmed with XTT. Then the scratch migration assay was used for assessing the wound healing. TRH was added to both control and hyperglycemia groups at $100 \mathrm{nM}$ after the scratch was created. The wound areas were measured after 24 and 48 hours after the scratch.

Results: TRH and/or hyperglycemia did not affect the cellular activity after 48 hours. TRH reduced the wound areas ( $42.6 \%$ ) compared to the control (52.2\%) after 24 hours. In the hyperglycemia group the wound area was $64.3 \%$ and $61.0 \%$ of initial area at the $24^{\text {th }}$ and $48^{\text {th }}$ hours respectively. TRH incubation reduced these wound areas to $55.2 \%$ and $47.1 \%$ of initial areas. Conclusion: TRH treatment accelerated wound healing in hyperglycemia, which indicates the positive effects of TRH in wounds, may occur in diabetes.
\end{abstract}

Keywords: Hyperglycemia, wound healing, scratch migration assay, fibroblast

\section{INTRODUCTION}

Skin protects the body like a barrier against external factors such as chemical substances, UV rays and infections. It also has an important role in homeostasis by regulating body temperature and fluid balance. Wound healing is a crucial process for the body. This process is extremely complex; many molecules and cells such as cytokines, chemokines, growth factors and inflammatory cells work together for healing (Hahm, Glaser, \& Elster, 2011). As a result of the problems that may develop during this process, wounds may become chronic. C hronic, non-healing wounds lead to pain, restlessness, recurrent infections and even amputation in progressive situations. Non-healing wounds have been shown to be one of the major causes of gangrene and septic deaths. Furthermore, industrialized countries spend more than 3\% of their health-care budgets on non-healing wounds (Ohnstedt, Lofton Tomenius, Vagesjo, \& Phillipson, 2019). Diabetes is one of the most common causes of non-healing wounds. In diabetic patients, even minor wounds are difficult to heal while susceptibility to infection increases. Amputations are performed in many diabetic patients, especially due to diabetic foot syndrome. Current symptomatic treatments are often insufficient for wound healing. Furthermore, more efficient treatments are expensive and long-term treatments reduce the quality of life. Therefore, new, efficient and inexpensive treatment choices are needed for non-healing wounds in diabetes.

Address for Correspondence:

Gökçen TELLi, e-mail: gokcentelli@hacettepe.edu.tr

This work is licensed under a Creative Commons Attribution 4.0 International License.
Submitted: 19.11 .2020

Revision Requested: 06.09.2021

Last Revision Received: 10.10 .2021

Accepted: 11.10 .2021

Published Online: 00.00 .0000 
The interaction between thyroid hormone and wound healing has been known and studied for many years (Zamick \& Mehregan, 1973). In euthyroid rats, thyroid treatment accelerated wound healing and, with supraphysiological doses of T4, increased the tensile strength of the skin (Lennox \& Johnston, 1973; Zamick \& Mehregan, 1973; Mehregan \& Zamick, 1974). However, after the expression of the hypothalamic-pituitary-thyroid axis components on the skin was reported (Slominski et al., 2002), studies about thyroid-wound healing issues started to increase again. Topical application of thyroid hormones accelerated wound healing for both normal and diabetic animals (Safer, Crawford, \& Holick, 2005; Kassem, Liberty, Babaev, Trau, \& Cohen, 2012; Tarameshloo, Norouzian, Zarein-Dolab, Dadpay, \& Gazor, 2012; Kaykhaei, 2016).

High levels of another important element of the thyroid axis, TRH, were also found in the skin (Slominski et al., 2002; Gaspar et al., 2010). Studies have shown that TRH has a remarkable role in hair growth and keratinocyte proliferation (Gaspar et al., 2010; Vidali et al., 2014). Meier et al. demonstrated that TRH promoted wound healing in frog and human skin in organ culture assays (Meier et al., 2013). TRH and its analogue taltirelin provided important wound healing in a primary rat fibroblast culture scratch test and also in an in vivo skin wound (Nie et al., 2014). Despite these remarkable results, the studies investigating the role of TRH on wound healing are very limited and the role of $\mathrm{TRH}$ on wound healing in diabetic conditions has not been studied. In this study; we investigated the possible effects of TRH on in vitro wound healing assays in both normoglycemic and hyperglycemic conditions.

\section{MATERIAL AND METHODS}

\section{Chemicals}

Low Glucose Dulbecco's Modified Eagle Medium (DMEM) was purchased from Wisent Bioproducts (Quebec, Canada). Fetal bovine serum (FBS) and trypsin -EDTA was purchased from Cegrogen-Biotech (Germany). L-glutamine and penicillinstreptomycin were purchased from Biochrom (Cambridge, UK). Sodium 3'-[1-[(phenylamino)-carbonyl]-3,4-tetrazolium]bis(4-methoxy-6-nitro)benzene-sulfonic acid hydrate (XTT) was purchased from PanReac Applichem (Germany). Phenazine methosulfate (PMS), glucose and mannitol were purchased from Sigma Aldrich (St Louis, USA). TRH Ferring 0.2 mg/ $\mathrm{ml}$ intravenous (Ferring Pharmaceuticals) was used in the experiments.

\section{Cell culture}

L929 mouse fibroblast cell line was used in the experiments. DMEM supplemented with 10\% FBS, 2\% L-glutamine and 1\% penicillin-streptomycin was used in all groups. In hyperglycemic groups, fibroblasts were incubated in DMEM medium containing 25 mM glucose for 48 hours (Ueck et al., 2017). Untreated fibroblasts which were incubated with only DMEM medium were the control group. In the hyperglycemia+TRH group, fibroblasts were incubated in DMEM containing $25 \mathrm{mM}$ glucose, and after 48 hours 100 nM concentration of TRH was added, in line with a previous study performed in fibroblasts
(Nie et al., 2014). In the TRH treatment group, fibroblasts were incubated in DMEM along with TRH (100 nM). In order to investigate the effects of osmolarity we used a mannitol treatment group with the same molarity as the hyperglycemia group. In the mannitol group, fibroblasts were incubated in DMEM with $20 \mathrm{mM}$ mannitol.

\section{XTT assay}

L929 cells were seeded into 96-well plates and incubated for 24 hours $\left(5 \% \mathrm{CO}_{2}, 37^{\circ} \mathrm{C}\right)$ to form a semi-confluent monolayer. After 24 hours of incubation, the medium was aspirated from the cells. All incubation conditions of different groups were mimicked. Cells were incubated for 48 hours $\left(5 \% \mathrm{CO}_{2}, 37^{\circ} \mathrm{C}\right)$. After 48 hours of treatment, $50 \mu$ l of the XTT/PMS solution was added to each well and the plates were further incubated for 3 to 5 hours in the incubator at $37^{\circ} \mathrm{C}$. Then the plates were swayed carefully and an aliquot of $100 \mu$ l was transferred from each well into the corresponding well of a new microplate and read at $450 \mathrm{~nm}$. (reference wavelength $630 \mathrm{~nm}$ ). The results were normalized to the control group.

\section{Scratch migration assay}

The migration of fibroblasts was analyzed by the scratch migration assay as previously described (Liang, Park, \& Guan, 2007; Nie et al., 2014). L929 cells were seeded in 24-well plates and maintained at $37^{\circ} \mathrm{C}$ and $5 \% \mathrm{CO}_{2}$ to permit cell adhesion and the formation of a confluent monolayer. After 48 hours, when $90 \%$ confluence was achieved, an artificial gap called a "scratch" approximately 0.2-0.4 mm in width, was created with a $200 \mu \mathrm{L}$ sterile pipette tip. The culture medium was then immediately removed (along with any detached cells and debris).

Cells were then incubated at the experiment conditions at $37^{\circ} \mathrm{C}$ and $5 \% \mathrm{CO}_{2}$ for 48 hours. The cell migration and morphological changes of cells were observed in the images taken by an inverted microscope, equipped with a digital camera. According to previous in vitro studies showing that TRH was effective at the $72^{\text {nd }}$ hour (Nie et al., 2014) and studies demonstrated that TRH provided faster wound healing (Meier et al., 2013) images were captured at three different time points (at the $0,24^{\text {th }}$ and $48^{\text {th }}$ hours). The width of the scratch and wound closure was analyzed by ImageJ 1.53 e software (National Institute of Health). Wound closure was quantified as wound area relative to the initial wound area.

\section{Statistical analysis}

Statistical analysis was performed with one-way ANOVA post hoc Tukey test. Data were represented as mean \pm standard error mean (SEM). The width of the scratch and wound closure was analyzed by ImageJ 1.53e software. GraphPad Prism 5.0 software was used for statistical analysis (San Diego, USA).

\section{RESULTS}

Firstly, the cell viability and proliferation were assessed with XTT. TRH incubation and/or hyperglycemia did not affect the cell after 48 hours (Figure 1). 


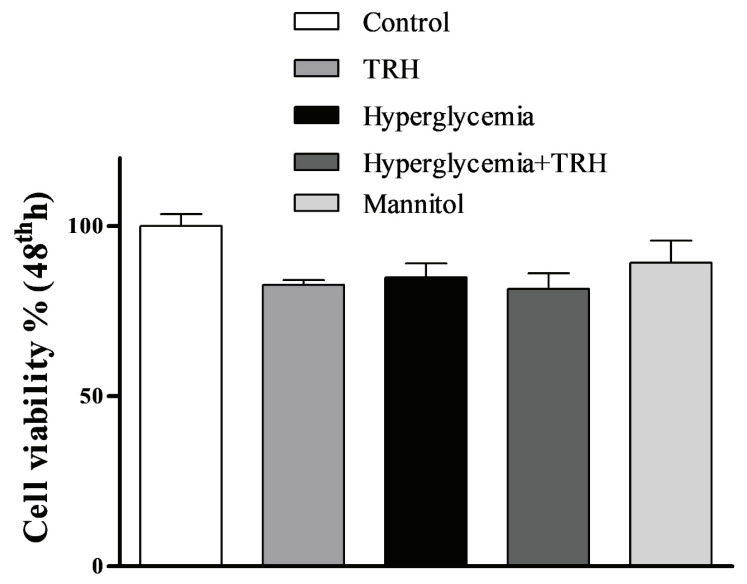

Figure 1. Cell viability \% of each group after 48 hours. Data were presented as mean \pm SEM. Statistical analysis was performed with one-way ANOVA, post hoc Tukey test. $\mathrm{n}=8$.

The scratch migration assay was used for assessing the wound healing. At the end of the $24^{\text {th }}$ hour, the TRH incubation to the normoglycemic cells reduced the wound area statistically significantly compared to that of the control group. The wound area was the greatest in the hyperglycemia group, which indicated that the hyperglycemia, as in diabetes mellitus, delayed wound healing in the L929 cell line. $100 \mathrm{nM} \mathrm{TRH}$ incubation accelerated the wound healing also in the hyperglycemia group and provided a significantly smaller wound area compared to the hyperglycemia group. The wound area in the mannitol group was not different from that of the control group, which showed that the differences in the wound areas between the groups were not related with hyperosmolarity (Figure 2).

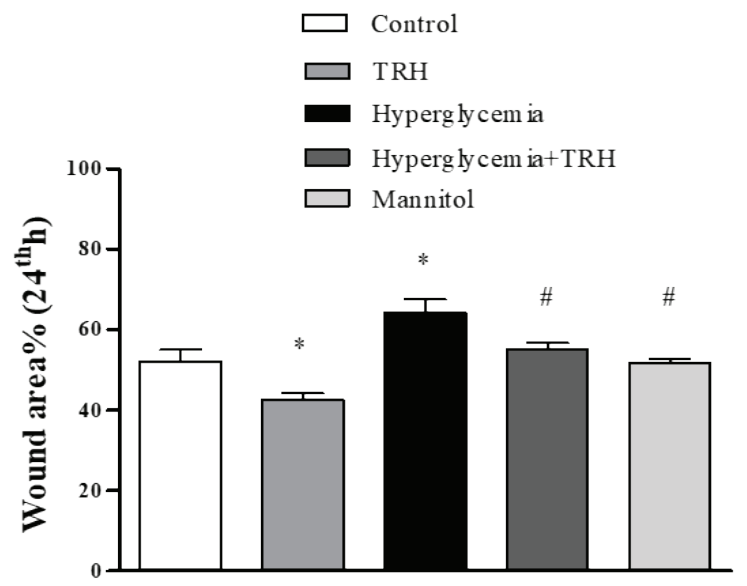

Figure 2. The wound areas of the groups at the end of the 24 hours. Data were presented as mean \pm SEM. Statistical analysis was performed with one-way ANOVA, post hoc Tukey test, ${ }^{*} p<0.05$ vs control and $\# p<0.05$ vs hyperglycemia group. $n=8$.

The 48-hour TRH incubation continued to reduce the wound area; however, this reduction was not different from that of the control group. On the other hand, in the hyperglycemic condition TRH incubation provided statistically significant healing in the wound area compared to the hyperglycemia group (Figure 3).

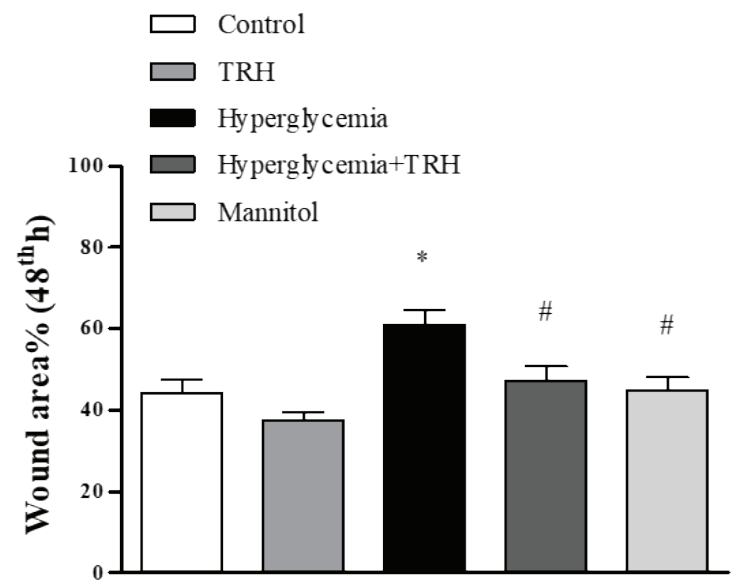

Figure 3. The wound areas of the groups at the end of the 48 hours. Data were presented as mean \pm SEM. Statistical analysis was performed with one-way ANOVA, post hoc Tukey test, ${ }^{*} p<0.05$ vs control and $\# p<0.05$ vs hyperglycemia group. $n=8$.

\section{DISCUSSION}

Recurrent, non-healing wounds are a serious problem in diabetic patients. Approximately $15 \%$ of these patients suffer from recurrent foot ulcers (Chun et al., 2019). The wounds that do not heal and become chronic affect quality of life and require high patient compliance. About 20\% to $33 \%$ of costs related to diabetes mellitus are used for treatments of diabetic foot ulcers (Schreml \& Berneburg, 2017). This situation affects the individuals and communities not only emotionally but also financially (Gouin \& Kiecolt-Glaser, 2011). The importance of more efficient choices in the treatment of wound healing will increase as indicated by the report notifying the increasing numbers of diabetic patients year by year ( $\mathrm{WHO}$, 2020).

Hyperglycemia affects many significant factors in the wound healing process (Pan et al., 2015; Leguina-Ruzzi \& Valderas, 2017). The fibroblasts under hyperglycemic conditions exhibited impaired function in previous studies (Lerman, Galiano, Armour, Levine, \& Gurtner, 2003). In wound healing, fibroblasts are critical cells that are responsible for the breakdown of fibrin clot, creating the collagen structures and extracellular matrix (Bainbridge, 2013). Therefore, fibroblast cells are very suitable targets for investigating the effects of new drugs or therapies in wound healing. Firstly, we showed that $100 \mathrm{nM} \mathrm{TRH}$, or the hyperglycemic condition created in cells, was not cytotoxic to the L929 cell line until the end of the experiments. However, the proliferation of the cells was only slightly reduced with hyperglycemia after 48 hours and TRH treatment did not provide an increase in the proliferation. There are conflicting results in the literature about this issue. In some of the experiments performed on various cells, a significant decrease was observed with high glucose administration (Gupta \& Tikoo, 2013), while high glucose did not change the proliferation in other cells (Morais, Westhuyzen, Pat, Gobe, \& Healy, 2005). In our study also there was no significant reduction in our experiments at the $48^{\text {th }}$ hour. However, considering the study that indicated the significant increase in proliferation of the primary fibro- 
blasts of rats with TRH after 72 hours, longer use of TRH may be required to increase the proliferation.

The scratch migration assay is a well-established, inexpensive and reproducible method that is used frequently in initial wound healing studies (Pitz Hdaet al., 2016). For these reasons, our study was designed in fibroblasts and the scratch migration assay was used as a wound healing test. Consistent with previous studies, hyperglycemia caused a delay in wound healing at both the $24^{\text {th }}$ and $48^{\text {th }}$ hours independent from hyperosmolarity in our study.

Today, once a wound occurs in the diabetic patient, off-landing, bandages and dressing that provides a moiety environment is used first (Everett \& Mathioudakis, 2018). However, the appropriate pharmacological interventions are essential for optimum healing. Many different effective antiseptics and topical antibiotics are used in the treatment in the first stage. However, with the recurrence of the wound these drugs can be inadequate. As a result, new and more powerful antibiotics, matrix metalloproteinase inhibitors, protease inhibitors, recombinant human platelet derived growth factor, and other new therapies such as negative pressure wound therapy and surgical processes are needed. Although more efficient results are obtained from these treatments, the very high cost of treatment and increased use of antibiotics are considered to be an important problem (Qi et al., 2020). Therefore, new, safer and cheaper treatments will be preferred for this challenging clinical problem. The repurposing of drugs has been frequently preferred to in recent years due to their reliability and more rapid usage in treatment (Abels \& Soeberdt, 2019). TRH has safely been used in thyroid diseases for many years. The efficient roles of TRH in skin elements such as keratinocytes, hair follicles and fibroblasts (Werner, Krieg, \& Smola, 2007) have directed investigators to wound healing. In an ex vivo study on frog skin that were showed that TRH provided significant reduction in the wound size 72 hours after the application and reached maximum at the $7^{\text {th }}$ day(Meier et al., 2013). The promising results were also obtained in the wound healing within days with TRH in the mice (Nie et al., 2014). Our study has taken it one step further and has demonstrated the efficiency of TRH on wound healing in hyperglycemic conditions. TRH treatment accelerated wound healing both 24 and 48 hours after the creation of the wound in hyperglycemic conditions, w hich may indicate that TRH may be beneficial for wound healing in diabetes mellitus.

There are many factors that delay wound healing in diabetes mellitus. It is thought that the increase in blood glucose level directly affects components in the wound healing process. Furthermore, neuropathic and biochemical abnormalities, vascular pathologies, reduced angiogenesis and impaired immune function may have effects on wound healing (Qi et al., 2020). It has been shown that TRH stimulated proliferation of immune cells and inhibited monocyte activity in nature killer cells in vitro (Frohlich \& Wahl, 2019). TRH injection reduced the elevated blood glucose levels in mice. In another study, thyrox- ine promoted angiogenesis of ex vivo wounded human skin cells (Zhang et al., 2019). Thus, it may be suggested that TRH can both directly and indirectly affect impaired functions due to hyperglycemia, and also provide accelerated wound healing in hyperglycemia.

The present study is the first that investigated the effect of $\mathrm{TRH}$ in wound healing under hyperglycemic conditions We also assessed the role of TRH under the normoglycemic conditions of L929 fibroblast cell line for the first time. Our results supported the previous studies investigating the effects of $\mathrm{TRH}$ on wound healing. Nie et. al reported direct effects of TRH on the alpha smooth muscle actin in fibroblasts and showed $\mathrm{TRH}$ increased proliferation and migration capacity of them (Nie et al., 2014). Similarly, in our study, after 24 hours of TRH treatment the wound area got smaller compared to the control group. However, after 48 hours there was no statistically significant difference between the control and TRH groups, even though TRH continued to be used to close the wound area. It could be related with the high proliferation capacity of cell lines that was used (Pitz Hda et al., 2016). Because of the fast migration of the cells in the control group, the healing that TRH provided could be masked. The earlier observation time can be preferred in future experiments. Wound healing is a very complex process, and it is known that keratinocytes are involved in this process as well as fibroblasts (Pastar, Stojadinovic, \& Tomic-Canic, 2008). Another limitation of our study is that evaluations were made only in fibroblasts, and future studies plan to evaluate the efficacy of TRH in keratinocytes. Thus, the mechanism of action of the role of $\mathrm{TRH}$ in wound healing will also be evaluated.

\section{CONCLUSIONS}

$\mathrm{TRH}$ indirectly regulates the thyroid hormone by stimulating TSH. Thyroid hormone has important physiological effects and affects many systems in the body. Therefore, our results demonstrate the direct effects of TRH and are promising for further studies and will guide in vivo experiments. TRH is an inexpensive and relatively safe drug that has been used for many years. The possible efficiency of TRH in impaired wound healing in diabetes will reduce health expenses caused by ulcers in diabetes. Because it is a drug that patients can easily access, the compliance of patients will increase. If our study will be confirmed by further in vivo studies a new treatment choice may be available.

Peer-review: Externally peer-reviewed.

Author Contributions: Conception/Design of Study- M.D., I.K., G.T.; Data Acquisition- M.D., I.K., G.T.; Data Analysis/InterpretationM.D., I.K., G.T.; Drafting Manuscript- M.D., I.K., G.T.; Critical Revision of Manuscript- M.D., I.K., G.T.; Final Approval and Accountability- M.D., I.K., G.T.

Conflict of Interest: The authors have no conflict of interest to declare.

Financial Disclosure: Authors declared no financial support. 


\section{REFERENCES}

- Abels, C., \& Soeberdt, M. (2019). Can we teach old drugs new tricks?-Repurposing of neuropharmacological drugs for inflammatory skin diseases. Experimental Dermatology, 28(9), 1002-1009. http://dx.doi.org.10.1111/exd.13987

- Bainbridge, P. (2013). Wound healing and the role of fibroblasts. Journal of Wound Care, 22(8), 407-408, 410-412. http://dx.doi. org.10.12968/jowc.2013.22.8.407

- $\quad$ Chun, D. I., Kim, S., Kim, J., Yang, H. J., Kim, J. H., ... Won, S. H. (2019). Epidemiology and Burden of Diabetic Foot Ulcer and Peripheral Arterial Disease in Korea. Journal of Clinical Medicine, 8(5). http:// dx.doi.org.10.3390/jcm8050748

- Everett, E., \& Mathioudakis, N. (2018). Update on management of diabetic foot ulcers. Annals of New York Academic Sciences, 1411 (1), 153-165. http://dx.doi.org.10.1111/nyas.13569

- Frohlich, E., \& Wahl, R. (2019). The forgotten effects of thyrotropin-releasing hormone: Metabolic functions and medical applications. Frontiers in Neuroendocrinolgy, 52, 29-43. http://dx.doi. org.10.1016/j.yfrne.2018.06.006

- $\quad$ Gaspar, E., Hardenbicker, C., Bodo, E., Wenzel, B., Ramot, Y., Paus, R. (2010). Thyrotropin releasing hormone (TRH): a new player in human hair-growth control. FASEB Journal, 24(2), 393-403. http:// dx.doi.org.10.1096/fj.08-126417

- Gouin, J. P., \& Kiecolt-Glaser, J. K. (2011). The impact of psychological stress on wound healing: methods and mechanisms. Immunology and Allergy Clinics of North America, 31 (1), 81-93. http:// dx.doi.org.10.1016/j.iac.2010.09.010

- Gupta, C., \& Tikoo, K. (2013). High glucose and insulin differentially modulates proliferation in MCF-7 and MDA-MB-231 cells. Journal of Molecular Endocrinology, 51(1), 119-129. http://dx.doi. org.10.1530/JME-13-0062

- Hahm, G., Glaser, J. J., \& Elster, E. A. (2011). Biomarkers to predict wound healing: the future of complex war wound management. Plastic and Reconstrative Surgery, 127 (Supp/ 1), 21S-26S. http:// dx.doi.org.10.1097/PRS.0b013e3181fbe291

- Kassem, R., Liberty, Z., Babaev, M., Trau, H., \& Cohen, O. (2012). Harnessing the skin-thyroid connection for wound healing: a prospective controlled trial in guinea pigs. Clinical and Experimental Dermatology, 37(8), 850-856. http://dx.doi.org.10.1111/j.13652230.2012.04456.x

- Kaykhaei, M. A., Shahraki, M. R., Mohammadi, M, Moshtaghian, B., Zandkarimi, M. (2016). The Effects of Topical Application of Thyroid Hormone (Liothyronine, T3) on Skin Wounds in Diabetic Wistar Rats. Zahedan Journal of Research in Medical Science., 18(4), e6449. http://dx.doi.org.10.17795/zjrms-6449

- Leguina-Ruzzi, A., \&Valderas, J. P. (2017). BLT2 expression improves skin integrity and protects from alterations caused by hyperglycemia in type 2 diabetes. Dermatoendocrinolgy, 9(1), e1267078. http://dx.doi.org.10.1080/19381980.2016.1267078

- Lennox, J., \& Johnston, I. D. (1973). The effect of thyroid status on nitrogen balance and the rate of wound healing after injury in rats. British Journal of Surgery, 60(4), 309. Retrieved from https:// www.ncbi.nlm.nih.gov/pubmed/4700245

- Lerman, O. Z., Galiano, R. D., Armour, M., Levine, J. P., \& Gurtner, G. C. (2003). Cellular dysfunction in the diabetic fibroblast: impairment in migration, vascular endothelial growth factor production, and response to hypoxia. Americal Journal of Pathology, 162(1), 303312. http://dx.doi.org.10.1016/S0002-9440(10)63821-7

- $\quad$ Liang, C. C., Park, A. Y., \& Guan, J. L. (2007). In vitro scratch assay: a convenient and inexpensive method for analysis of cell migration in vitro. Nature Protocols, 2(2), 329-333. http://dx.doi.org.10.1038/ nprot. 2007.30
Mehregan, A. H., \& Zamick, P. (1974). The effect of triiodothyronine in healing of deep dermal burns and marginal scars of skin grafts. A histologic study. Journal of Cutanaeous Pathology, 1(3), 113-116. http://dx.doi.org.10.1111/j.1600-0560.1974.tb00201.x

Meier, N. T., Haslam, I. S., Pattwell, D. M., Zhang, G. Y., Emelianov, V., .. Paus, R. (2013). Thyrotropin-releasing hormone (TRH) promotes wound re-epithelialization in frog and human skin. PLOS One, 8(9), e73596. http://dx.doi.org.10.1371/journal.pone.0073596

Morais, C., Westhuyzen, J., Pat, B., Gobe, G., \& Healy, H. (2005). High ambient glucose is effect neutral on cell death and proliferation in human proximal tubular epithelial cells. American Journal of Physiology Renal Physiology, 289(2), F401-409. http://dx.doi. org.10.1152/ajprenal.00408.2004

Nie, C., Yang, D., Liu, N., Dong, D., Xu, J., \& Zhang, J. (2014). Thyrotropin-releasing hormone and its analogs accelerate wound healing. Journal of Surgical Research, 189(2), 359-365. http://dx.doi. org.10.1016/j.jss.2014.03.004

Ohnstedt, E., Lofton Tomenius, H., Vagesjo, E., \& Phillipson, M. (2019). The discovery and development of topical medicines for wound healing. Expert Opinion in Drug Discovery, 14(5), 485-497. http://dx.doi.org.10.1080/17460441.2019.1588879

Pan, F., Guo, R., Cheng, W., Chai, L., Wang, W., .. Li, S. (2015). High glucose inhibits $\mathrm{ClC}-2$ chloride channels and attenuates cell migration of rat keratinocytes. Drug Design Development and Therapy, 9, 4779-4791. http://dx.doi.org.10.2147/DDDT.S84628

Pastar, I., Stojadinovic, O., \& Tomic-Canic, M. (2008). Role of keratinocytes in healing of chronic wounds. Surgical Technology International, 17, 105-112. Retrieved from https://www.ncbi.nlm.nih. gov/pubmed/18802889

Pitz, H. S., Pereira, A., Blasius, M. B., Voytena, A. P., Affonso, R. C., . . Maraschin, M. (2016). In Vitro Evaluation of the Antioxidant Activity and Wound Healing Properties of Jaboticaba (Plinia peruviana) Fruit Peel Hydroalcoholic Extract. Oxidative Medicine and Cellular Longevity, 2016,3403586. http://dx.doi.org.10.1155/2016/3403586 - $\quad$ Qi, L., Ahmadi, A. R., Huang, J., Chen, M., Pan, B., .. Sun, Z. (2020). Major Improvement in Wound Healing Through Pharmacologic Mobilization of Stem Cells in Severely Diabetic Rats. Diabetes, 69(4), 699-712. http://dx.doi.org.10.2337/db19-0907

- Safer, J. D., Crawford, T. M., \& Holick, M. F. (2005). Topical thyroid hormone accelerates wound healing in mice. Endocrinology, 146(10), 4425-4430. http://dx.doi.org.10.1210/en.2005-0192

- $\quad$ Schreml, S.,\& Berneburg, M. (2017). The global burden of diabetic wounds. British Journal of Dermatology, 176(4), 845-846. http:// dx.doi.org.10.1111/bjd.15254

Slominski, A., Wortsman, J., Kohn, L., Ain, K. B., Venkataraman, G. M., ... Tobin, D. J. (2002). Expression of hypothalamic-pituitarythyroid axis related genes in the human skin. Journal of Investigative Dermatology, 119(6), 1449-1455. http://dx.doi.org.10.1046/ j.1523-1747.2002.19617.x

Tarameshloo, M., Norouzian, M., Zarein-Dolab, S., Dadpay, M., \& Gazor, R. (2012). A comparative study of the effects of topical application of Aloe vera, thyroid hormone and silver sulfadiazine on skin wounds in Wistar rats. Laboratory Animal Research, 28(1), 1721. http://dx.doi.org.10.5625/lar.2012.28.1.17

- Veck, C., Volksdorf,T., Houdek, P., Vidal, Y. S. S., Sehner, S., ... Brandner, J. M. (2017). Comparison of In-Vitro and Ex-Vivo Wound Healing Assays for the Investigation of Diabetic Wound Healing and Demonstration of a Beneficial Effect of a Triterpene Extract. PLoS One, 12(1), e0169028. http://dx.doi.org.10.1371/journal.pone.0169028

- Vidali, S., Knuever, J., Lerchner, J., Giesen, M., Biro, T., . . Paus, R. (2014). Hypothalamic-pituitary-thyroid axis hormones stimulate mitochondrial function and biogenesis in human hair follicles. Journal of Investigative Dermatolgy, 134(1), 33-42. http://dx.doi. org.10.1038/jid.2013.286 
Istanbul J Pharm 51 (3): 313-318

- Werner, S., Krieg, T., \& Smola, H. (2007). Keratinocyte-fibroblast interactions in wound healing. Journal of Investigative Dermatolgy, 127(5), 998-1008. http://dx.doi.org.10.1038/s.j.jid.5700786

- WHO. (2020). Diabetes, Key facts. Retrieved from https://www. who.int/news-room/fact-sheets/detail/diabetes

- Zamick, P., \& Mehregan, A. H. (1973). Effect of 1-tri iodothyronine on marginal scars of skin grafted burns in rats. Plastic and Reconstructive Surgery, 51(1), 71-75. http://dx.doi.org.10.1097/00006534197301000-00013
- Zhang, G. Y., Langan, E. A., Meier, N. T., Funk, W., Siemers, F., \& Paus, R. (2019). Thyroxine (T4) may promote re-epithelialization and angiogenesis in wounded human skin ex vivo. PLoS One, 14(3), e0212659. http://dx.doi.org.10.1371/journal.pone.0212659 